\title{
Real-Time Dosimetry for Prostate Brachytherapy Using TRUS and Fluoroscopy
}

\author{
Danny French ${ }^{1}$, James Morris ${ }^{2}$, Mira Keyes $^{2}$, and S.E. Salcudean ${ }^{1}$ \\ 1 Department of Electrical and Computer Engineering, University of British \\ Columbia, 2356 Main Mall, Vancouver BC V6T 1Z4, Canada \\ 2 Vancouver Center, British Columbia Cancer Agency, 600 West 10th, Vancouver, \\ BC V5Z 4EB, Canada
}

\begin{abstract}
A new approach for computing real-time dosimetry (RTD) for prostate brachytherapy is presented. Transrectal ultrasound (TRUS) and fluoroscopic images are fused to compute dosimetry. The frontal plane coordinates of the seeds are found in the fluoroscopic images and the remaining coordinate is computed from the TRUS. Our approach is verified on a phantom and tested on clinical data. A method for tracking intraoperative seed motion using TRUS is also presented.
\end{abstract}

\section{Introduction}

Prostate brachytherapy is frequently used to treat low-risk prostate cancer. Using transrectal ultrasound (TRUS) to guide needles between 80 and 150 small radioactive seeds are permanently implanted in the prostate.

Because of inaccuracies in needle placement and intraoperative prostate shifting and swelling, there is a need to provide accurate real-time dosimetric feedback. This feedback will allow radiation oncologists to intraoperatively modify the planned seed locations (i.e. interactive planning) ensuring the prostate receives sufficient radiation to destroy the cancerous cells [1] [2].

The need for real-time dosimetry (RTD) has resulted in several commercial systems. VariSeed (Varian Medical Systems, Palo Alto, CA), Prostate Implant Planning Engine for Radiotherapy (PIPER) system (RTek, Pittsford, NY), Interplant System (Burrdette Medical System, Champaign, IL), Strata System (Rosses Medical Systems, Columbia, MD), and SPOT (Nucletron Corporation, Veenandaal, Netherlands) all use ultrasound to locate needles or blood trails and assume seed spacing to compute dosimetry. Individual seed are not located because seeds cannot be reliably located in ultrasound images [7. Therefore, another modality must be combined with TRUS to accurately compute dosimetry.

Because fluoroscopes are commonly used, in addition to TRUS, for prostate brachytherapy, several methods of fusing TRUS and fluoroscopic images to compute dosimetry have been reported. TRUS is used to identify the prostate contour and fluoroscopic images taken from three or more perspectives are used to locate individual seeds to compute dosimetry. In 3. TRUS and three fluoroscopic images are fused using four needle tips. In [5] the fluoroscopic images are registered to the TRUS image using five to seven noncoplanar reference points. Yet, 
interactive planning is limited because the fluoroscope must be rotated to three different angles to update dosimetry, which is both time-consuming and inconsistent with the current procedure. A RTD system using intraoperative magnetic resonance (IMR) imaging is presented in [4]. This method cannot consistently locate individual seeds and is expensive.

This paper presents a new approach for RTD in prostate brachytherapy. Our system is fast enough to allow interactive planning and integrates into the current procedure with minimal change and without additional imaging equipment. TRUS is used to locate each needle tip and a single fluoroscopic image is used to locate individual seeds. The fluoroscopic image frame and TRUS image frame are registered, using a single fluoroscopic image of the TRUS probe. Knowing the location of each seed, dosimetry can be computed and displayed after each fluoroscopic image providing the radiation oncologist with the ability to confidently make modifications to the preoperative plan. A method of compensating for intraoperative seed motion is also presented.

\section{TRUS and Fluoroscopic Based RTD - Methods}

A flow chart of our approach is shown in Fig. 1. The coordinate systems used are described in Fig. 2a. Initially, as is current procedure at the Vancouver Cancer Center, the radiation oncologist positions the TRUS probe based on manual registration of preoperative TRUS images with intraoperative TRUS images. Once this is achieved, a single fluoroscopic image of the TRUS probe is used to register the fluoroscopic and TRUS images. The radiation oncologist continues with the current procedure by inserting each needle and locating its tip in the TRUS image (see Fig. 2b). From the tip location and the entry point of the needle, a needle path is computed by interpolating from the needle base to the tip. After each row of needles, a fluoroscopic image is taken to determine the $(\mathrm{x}, \mathrm{z})$-coordinates of the seeds (see Fig. 25). Using these coordinates and the needle path, the y-coordinates of the seeds are computed. Knowing the coordinates of each seed allows dosimetry to be displayed to the radiation oncologist after each row of needles, so the preoperative plan can be intraoperatively modified to reflect inaccuracies in seed placement and intraoperative seed motion.

Registration of TRUS and Fluoroscopic Images. The TRUS and fluoroscopic images are registered using a single fluoroscopic image of the TRUS probe. The edges of the probe are found in the fluoroscopic image using an intensitybased edge detector and a least squares fit. Knowing the corners of the probe in the fluoroscopic image, the physical dimensions of the probe and the source to image plane distance of the C-arm, a simple coordinate transform from the fluoroscopic image to the TRUS image can be found using similar triangles.

Fig. 3 shows the error in determining the height of the TRUS as a function of fluoroscopic image resolution. The accuracy of the height of an object above the image plane of the fluoroscope is primarily limited by the resolution of the 


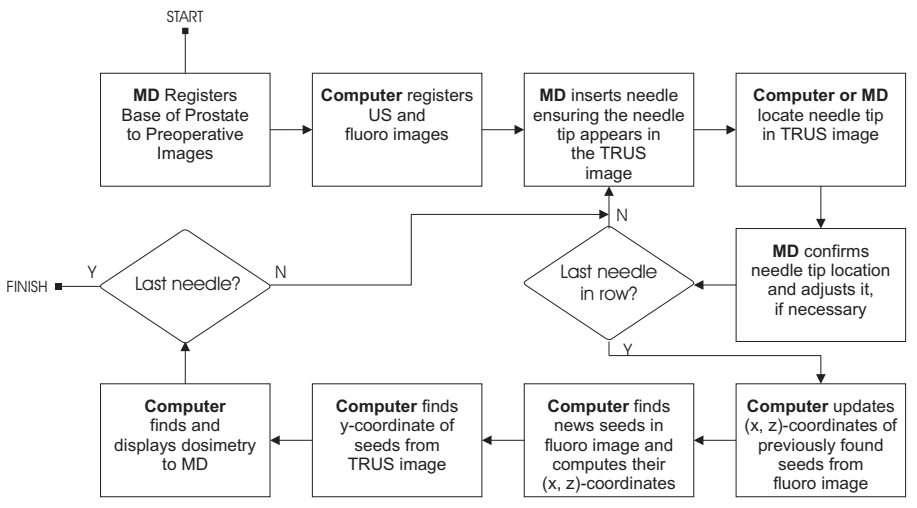

Fig. 1. A flow chart of our approach for computing RTD

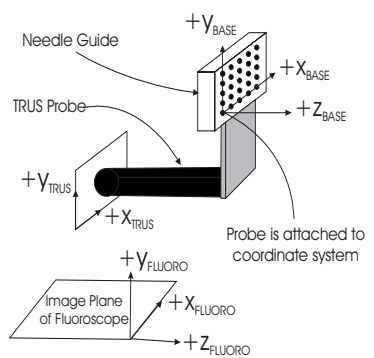

(a)

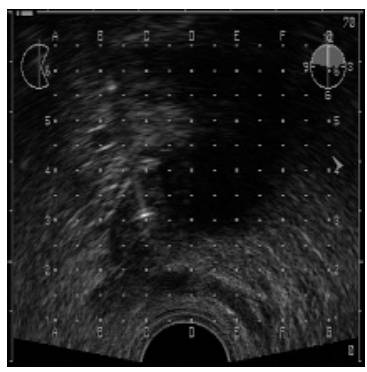

(b)

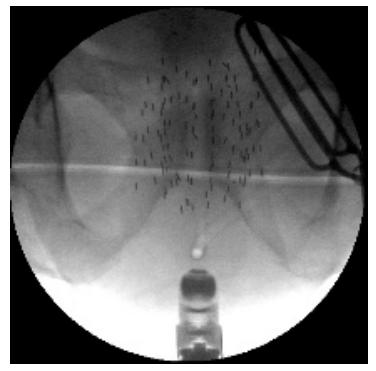

(c)

Fig. 2. (a) The coordinate systems used in our approach, (b) A TRUS image with needle artifact at C3, (c) A fluoroscopic image of implanted seeds

fluoroscope. While fluoroscopic image warping does contribute to error, it can be calibrated out 8 . In the data presented, image warping is ignored as it is not a determining factor in proving the feasibility of our approach. For simplicity, we assume that the C-arm and TRUS frames are parallel. The angular offsets between frames are small and contribute minimal errors. If necessary, they can be computed from the angles of edges that are known to be parallel.

The most significant error is in determining the height of the TRUS above the fluoroscopic image. Experimentally, the height of the TRUS was found using our registration algorithm for 15 different heights, with a mean absolute error of $2.16 \mathrm{~mm}$ and a maximum absolute error of $5.72 \mathrm{~mm}$.

Locating the Needle Tip in TRUS Images. The needle tip artifact is a white flash in the TRUS image (see Fig. 2b). Currently, this is manually located in the TRUS image, but work is underway to automate this step. By doing 


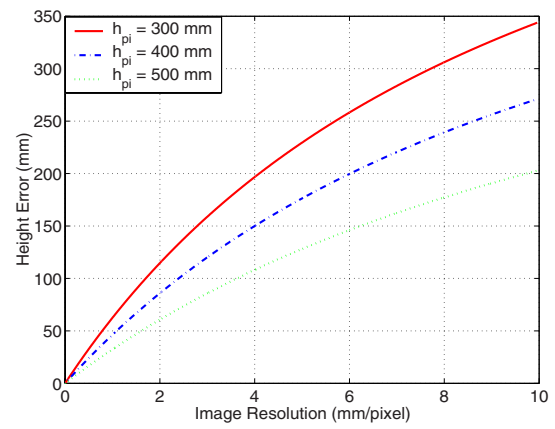

(a)

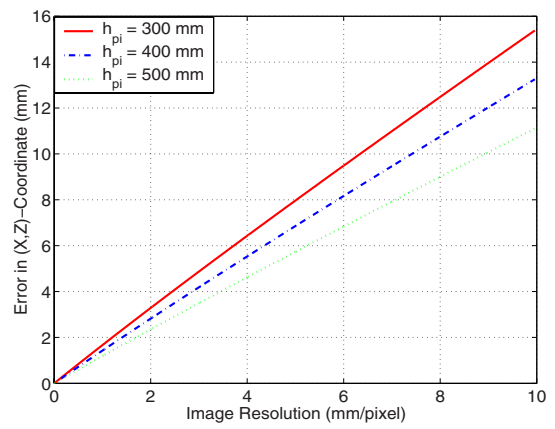

(b)

Fig. 3. (a) TRUS height error as a function of fluoroscopic image resolution, (b) Error in the $(\mathrm{x}, \mathrm{z})$-coordinates of the seeds as a function of image resolution

a localized search of sequential frames the location of the needle tip can be approximated by finding a sudden clustered change in intensity between frames. However, identifying the exact location of the needle tip from the needle artifact is difficult [3]. This is why a manual step for locating the needle tip is included in Fig 1

(X,Z)-Coordinates of Seeds from a Fluoroscopic Image. Because the height of the probe can be approximated reasonably well, fluoroscopic images can be back-projected to the TRUS frame to determine the (x,z)-coordinates of the seeds. Fig. $3 \mathrm{~b}$ shows the error in the (x,z)-coordinates as a function of image resolution with an accuracy of less than $0.75 \mathrm{~mm}$. The seeds are currently selected manually, but automation of this step using morphological operators has been demonstrated in [6], [5] and [3].

Because the seeds move, the intraoperative (x,z)-coordinates of the seeds must be updated with each fluoroscopic image. By searching in the regions near the coordinates of the previously found seeds, the seed coordinates can be updated. This step is done prior to searching for new seeds to simplify the search.

Y-Coordinate of Seeds from TRUS Images. It is obvious from Fig. 3a that the height of the seeds cannot be found from a single fluoroscopic image because of limited fluoroscopic image resolution (approximately $0.5 \mathrm{~mm} / \mathrm{pixel}$ ). In fact, even with a substantially higher resolution height errors are significant. However, the needle tip can be located in TRUS with much higher accuracy. So, knowing the entry point of the needle from the preoperative plan, a needle path is interpolated and the $\mathrm{y}$-coordinates of the seeds calculated. 
Computation of Dosimetry. Knowing the coordinates of each seed the dosimetry can be calculated. In this paper, each seed is modelled as a line source. The dosimetry results are displayed in the base frame which can easily be superimposed on TRUS images. This method of displaying dosimetry does not require the time-consuming step of segmenting the prostate, but allows the radiation oncologist to identify underdosed regions to do interactive planning.

\section{TRUS and Fluoroscopic Based RTD - Experiments}

\subsection{Phantom}

The phantom shown in Fig. 4a was constructed to validate the registration algorithm and to estimate the accuracy with which our system can locate seeds. The phantom is contained in a Plexiglas box (approximately $100 \mathrm{~mm}$ in each dimension). In one wall of the box there is one $32.5 \mathrm{~mm}$ diameter hole to insert a TRUS probe and four $1.5 \mathrm{~mm}$ diameter holes to insert needles. Mounted on the inside of the same wall is a metal needle guide to prevent needle deflection. On the inside of the opposite wall there is a Plexiglas shelf with four groves to hold seeds that are aligned with the holes in the needle guide. A latex condom filled with ultrasound gel extends from the $32.5 \mathrm{~mm}$ diameter hole to the opposite wall to simulate the rectum. Twelve seeds are secured in the groves of the Plexiglas shelf and the remaining space in the phantom is filled with gelatin (13 percent gelatin and 3 percent cellulose by mass).

A TRUS probe was inserted in the condom to a pre-determined depth between the metal needle guide and the Plexiglas shelf (the angle was measured to be approximately zero). A single fluoroscopic image was acquired (see Fig. 4b). The first needle was inserted until the needle tip appeared in the TRUS image (see Fig. 4c). The distance from the wall of the phantom to the hub of the needle was recorded. Using this distance and the fluoroscopic image of the TRUS probe, the TRUS image and fluoroscopic image spaces were registered. Three more needles were inserted until each needle tip appeared in the TRUS image.

The seed coordinates found by our system closely match the known seed locations. The mean error in the $\mathrm{x}, \mathrm{y}, \mathrm{z}$ directions is $0.47 \mathrm{~mm}, 0.52$ and $0.78 \mathrm{~mm}$, respectively, and the maximum error is $1.13 \mathrm{~mm}, 0.63 \mathrm{~mm}$ and $1.38 \mathrm{~mm}$. These results support our approach for registering the TRUS and fluoroscopic image and our method for computing the coordinates of the seeds.

\subsection{Clinical Data}

Our approach was tested on clinical data collected during a prostate brachytherapy procedure. The ultrasound video was captured at 30 frames per second and fluoroscopic images were taken as described in Fig. 1. Both the seeds in the fluoroscopic images and the needle tips in the TRUS images were manually selected, but the feasibility of our approach is still proven. The results of our system are compared to the seed locations and dosimetry found from a computed tomography (CT) scan done 3-4 hours after the procedure. 


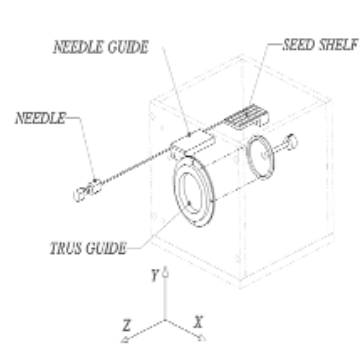

(a)

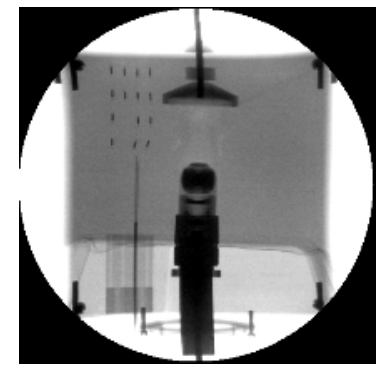

(b)

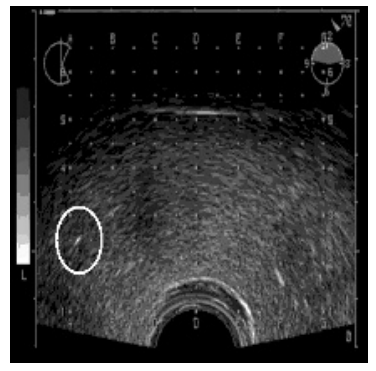

(c)

Fig. 4. (a) Diagram of the phantom, (b) Fluoroscopic image of the phantom, (c) TRUS image of the phantom (a needle artifact is enclosed by a circle)

Dosimetry was calculated for $3 \mathrm{~mm}^{3}$ voxels for seed locations determined from the CT scan and our approach. Using CT as the gold standard, the error in dosimetry for each voxel was computed. The mean error was 14.4 percent with a standard deivation of 3.8 percent. The percent volume is plotted as a function of percent error in dosimetry in Fig. 6a.

Possible sources of error include lack of calibration and dewarping, errors in manually selecting seeds and needle tips, and errors in locating seeds in the CT images. However, a significant amount of error results from not compensating for intraoperative seed motion in the $\mathrm{y}$-direction, which results from patient motion, probe motion and intraoperative prostate swelling and shifting.

\section{Tracking Intraoperative Seed Motion}

To compensate for motion in the $y$-direction, the motion of the prostate boundary or several seeds or blood trails is tracked in TRUS. Although seeds and blood trails cannot be consistently located or distinguished in TRUS, several image artifacts or the boundary of the prostate can be marked and cropped from a previous TRUS frame. The masks are correlated with a larger region having the same centroid in the new frame. The location of the maximum correlation coefficient is the new location of the mask. A weighted average based on the distance between the masks and the seeds is used to determine the vertical motion of each seed.

This seed tracking algorithm was also tested on clinical data. During the procedure the height of the probe was adjusted, the frames immediately before and after the adjustment are shown as Figs. 5 a and 5b. The location of three seeds or blood trails was found in both the old and new frames, by finding the centroid of the artifact using a thresholding technique. Two seeds or blood trails and the posterior boundary of the prostate were selected as masks (see 
Table 1. The results of tracking seed motion in clinical data

\begin{tabular}{|c|c|c|}
\hline Seed & $\begin{array}{c}\text { Motion in } \\
\text { Image }(\mathrm{mm})\end{array}$ & $\begin{array}{c}\text { Detected Seed } \\
\text { Motion }(\mathrm{mm})\end{array}$ \\
\hline 1 & -0.924 & -0.947 \\
\hline 2 & -0.924 & -0.912 \\
\hline 3 & -0.922 & -0.995 \\
\hline
\end{tabular}

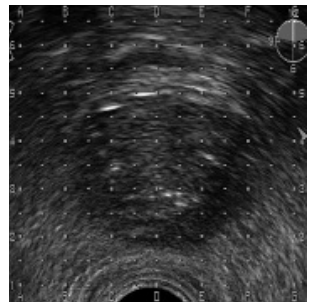

(a)

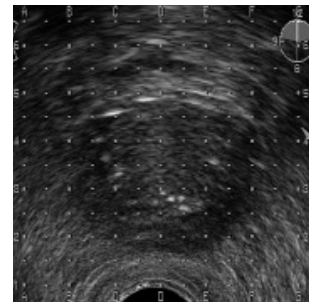

(b)

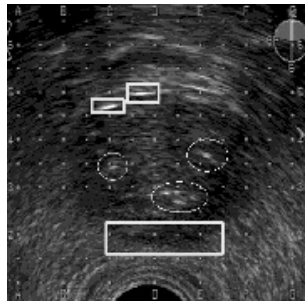

(c)

Fig. 5. (a) The old frame, (b) The new frame (note seed motion between frames), (c) The masks (enclosed in rectangles) and seeds (circled) used to verify the algorithm in clinical table

Fig. 55. The motion detected by our algorithm was compared to the motion of the centroid of each seed or blood trail artifact. The results in Table 1 show that seed motion can be intraoperatively tracked in TRUS images of the prostate.

The dosimetry was also calculated for the same data presented in Section 3.2 , with compensation for intraoperative seed motion. The mean error in dose was 11.2 percent with a standard deviation of 4.5 percent. The percent volume is plotted as a function of percent error in dosimetry in Fig. 6b.

In seed distributions that are compensated for motion in the y-direction the top rows of seeds more closely match the seed distribution found from the CT data. In our current system, masks are manually selected by the user, but work is ongoing to automate this process and determine the best choice of masks. It has been noted that TRUS frames recorded during a needle insertion cannot reliably identify seed motion because the needle forces temporarily cause motion beyond the actual resting point of the prostate.

\section{Conclusion and Future Work}

A new approach for computing RTD for prostate brachytherapy has been presented. The systems accounts for inaccuracies in needle placement and intraoperative seed motion. Dosimetry can be determined after each fluoroscopic image providing real-time dosimetric feedback and allowing interactive planning. 
As this is a work in progress, improvements in automation and accuracy are possible. The best approach to selecting masks to track intraoperative seed motion needs to be determined. The accuracy with which the needle tip can be found in a TRUS image must be validated. Our approach could be better validated by comparing our results to the seed locations found from three fluoroscopic images, rather than seed locations determined from a CT scan.

The approach has been validated on a phantom and tested on clinical data with promising results.

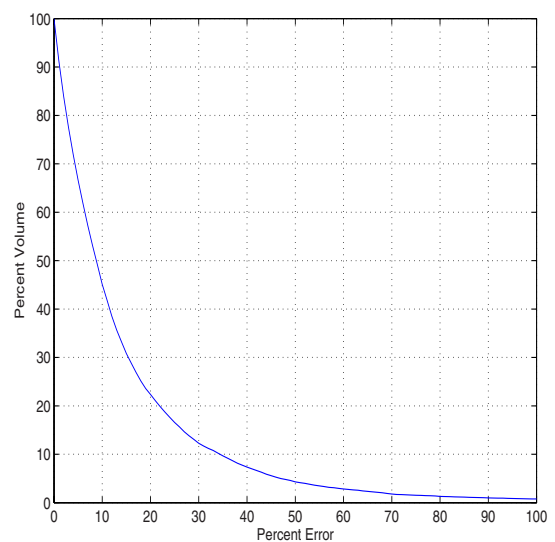

(a)

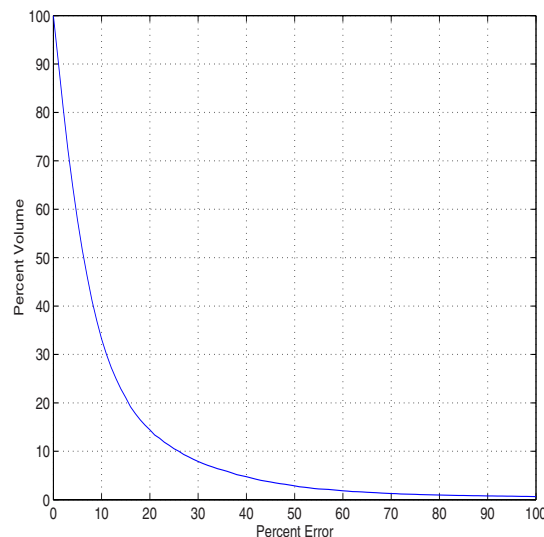

(b)

Fig. 6. The percent volume as a function of percent error in dosimetry without (a) and with (b) motion compensation for the y-coordinates of the seeds

\section{References}

[1] Subir Nag, Jay P. Ciezki, Robert Cormack, et al. Intraoperative planning and evalutation permanent prostate brachytherapy: Report of the American Brachytherapy Society. Int. J. Radiation Oncology Biol Phys, 51:1422-1430, 2001.

[2] L. Potter. Permanent prostate brachytherapy in men with clinically localised prostate cancer. Clinical Oncology, 15:301-315, 2003.

[3] L. Gong, P.S. Cho, B.H. Han, K.E. Wallner, et al. Ultrasonography and fluoroscopic fusion for prostate brachytherapy dosimetry Int. J. Radiation Oncology Biol Phys, 54:1322-1330, 2002.

[4] R.A. Cormack, H. Kooy, C.M. Tempany, A.V. D'Amico. A clinical method for real-time dosimetric guidance of transperineal ${ }^{125} i$ prostate implants using interventional magnetic resonance imaging. Int. J. Radiation Oncology Biol Phys, 46:207-214, 2000 . 
[5] D.A. Todor, M. Zaider, G.N. Cohen, et al. Intraoperative dyanmic dosimetry for prostate implants. Physics in Medicine and Biology, 48:1153-1171, 2003.

[6] D. Tubic, A. Zaccarin, J. Pouliot, and L. Beaulieu. Automated seed detection and three-dimensional reconstruction I: Seed locatization from fluoroscopic images or radiographs. Physics in Medicine and Biology, 28:2265-2271, 2001.

[7] B.H. Han, K. Wallner, G. Merrick, et al. Prostate brachytherapy seed identification on post-implant TRUS images Medical Physics, 30:898-900, 2003.

[8] G. Gil and Y. Yoon. C-arm image distortion calibration method for computeraided surgery. International Journal of Human-friendly Welfare Robotic Systems, 4:20-26, 2003. 